\title{
Germanica
}

\section{Peter Turrini und die avantgardistische Erneuerung des Volksstücks in Österreich}

Peter Turrini ou le renouveau avant-gardiste du théâtre populaire autrichien

\section{Alfred Strasser}

\section{OpenEdition}

\section{Journals}

Édition électronique

URL : http://journals.openedition.org/germanica/2220

DOI : $10.4000 /$ germanica.2220

ISSN : 2107-0784

Éditeur

Université de Lille

Édition imprimée

Date de publication : 1 décembre 1994

Pagination : 137-147

ISSN : 0984-2632

Référence électronique

Alfred Strasser, "Peter Turrini und die avantgardistische Erneuerung des Volksstücks in Österreich », Germanica [Online], 14 | 1994, Online erschienen am: 20 Januar 2014, abgerufen am 06 Oktober 2020 URL : http://journals.openedition.org/germanica/2220 ; DOI : https://doi.org/10.4000/germanica.2220

Ce document a été généré automatiquement le 6 octobre 2020.

(c) Tous droits réservés 


\title{
Peter Turrini und die avantgardistische Erneuerung des Volksstücks in Österreich
}

Peter Turrini ou le renouveau avant-gardiste du théâtre populaire autrichien

\author{
Alfred Strasser
}

ES GEHT ZU ENDE !!!

Zu ENDE mit den Worten der schönen Kunst, der erwählten Literatur, diesem Dreck auf dem schmackhaften Butterbrot einer verlorenen Sensibilität.

Zu ENDE mit den Worten der Werbung, der Büroklammern der Sprache, die als Nägel von den Fingern des Wohlstandes gerissen werden: AAUUUAAAA schreit

ELISABETH ARDEN.

Zu ENDE mit der Sprache der Ideologien, Programme, Ismen, diesem zähflüssigen Pesthauch in der humanistischen Schweinsblase des Bürgers.

$\mathrm{Zu}$ ENDE mit den Worten der Liebe, diesen scheißigen und soufflierten EKG-Tönen der

Sprache.

$\mathrm{Zu}$ ENDE mit diesem Brief. Am ANFANG war das Grunzen. Und so soll es werden. Und so soll es bleiben. BH von TRIUMPF! driumpfff! driumpff!! druuummmffffff uummmpfffff !!!!!!! uuuuuuuuuummmmmmmmpffffffff !!!!! umpf! ${ }^{1}$ 
1 Diese Zeilen schrieb 1971 der junge Peter Turrini an den Luchterhand-Verlag. Der damals siebenundzwanzigjährigefrühere Werbespottexter - einer von vielen Berufen hatte gerade mit seinem Bühnenerstling «Rozznjogd» einen durchschlagenden Erfolg und beendete sein zweites Stück «Sauschlachten». Ein neuer Vertreter des «Kritischen Volksstücks» ${ }^{2}$, das Ende der 60er Jahre im Zuge der Reflexion über das Verhältnis von Literatur und Realität, die besonders das Medium «Sprache» als Thema hatte ${ }^{3}$, entsteht, tritt an die Öffentlichkeit. Er formuliert das Problem der Sprachlosigkeit, dem FetischThema der österreichischen Literatur in den sechziger und siebziger Jahren, am radikalsten, indem er von der Position ausgeht, daß der Mensch zu keiner anderen authentischen Ausdrucksweise als zum Grunzen fähig sei, ohne die Entwicklungsfähigkeit hin zum Erwerb einer Sprache, die Kommunikation ermöglicht.

2 Exemplarisch will ich diese provokante Position an einem seiner Theaterstücke untersuchen; ich habe «Die Minderleister» gewählt, nicht zuletzt, weil es einen unmittelbaren Zeitbezug zu einer sozialen Problematik hat.

\section{I}

3 Turrinis Stück «Die Minderleister» wurde am 1. Juni 1988 im Wiener Akademietheater uraufgeführt und erschien ein Jahr darauf in Buchform. Der Anlaß für das Stück ist ein sehr konkreter, nämlich die Krise in der österreichischen Stahlindustrie in den 80er Jahren und die sich daraus ergebenden Entlassungen in den Werken der VOEST in Linz und in der Obersteiermark. 1980 waren in der Stahlindustrie noch 413752 Personen beschäftigt, 1990 waren es nur mehr 377 130, also fast zehn Prozent weniger. Turrini liegt diese Branche besonders am Herzen, weil sie am härtesten von der allgemeinen Wirtschaftskrise betroffen ist, und er einen persönlichen Bezug zu ihr hat: Er war selber 1962-63 in der VOEST in Linz beschäftigt. Dazu, wie er zu diesem Thema kam, erzählt Turrini folgendes:

In den Personalbüros der Stahlwerke liegen Listen mit den Namen von Arbeitern, die in nächster Zeit entlassen werden. Auf den Listen steht das Wort «Minderleister». Mit diesem Wort verbinde ich den schäbigen Versuch, die Krise in der Stahlindustrie auf Kosten der Schwächsten auszutragen. Angesichts der Arbeit, die diese Menschen in den Stahlwerken vollbringen, ist dieser Begriff eine besondere Ironie. Die Herstellung von Stahl läßt eine «Minderleistung» überhaupt nicht $\mathrm{zu}^{4}$

4 Am Stahlarbeiter Hans versucht nun Turrini exemplarisch die Konsequenzen einer Entlassung aufzuzeigen. Hans, Hilfsarbeiter in einem Stahlwerk, versucht durch Fortbildungskurse bester Arbeiter zu werden. Nichtsdestotrotz legt man ihm eines Tages nahe zu kündigen, was er aber nicht macht. Kurze Zeit später wird er bei der ersten sich bietenden Gelegenheit fristlos entlassen. Nachdem er beim Arbeitsminister vorgesprochen hat, wird er wieder eingestellt - diesmal als Ordner. Sein materielles Leben scheint gesichert, aber er wird von den Arbeitskollegen gemieden. Gänzlich isoliert, stürzt er sich in einen Hochofen ${ }^{5}$.

5 Als Nebenhandlung wird auch das Schicksal von Hans' Frau Anna gezeigt. Sie verliert ebenfalls ihre Arbeit in einer Schuhfabrik, weil man diese in ein Billiglohnland verlegt. Um ihren Lebensunterhalt zu verdienen, wird sie Darstellerin in einem Billigpornofilm.

6 Der melodramatische Opfertod von Hans mach deutlich, daß es Turrini bei der Darstellung seiner Arbeiter in erster Linie nicht um das Aufzeigen ökonomischer 
Mechanismen geht, sondern um ein ethisches Problem. Nicht die Gründe für die Entlassung werden gezeigt, sondern die Konsequenzen einer solchen - ein Martyrium für den Entlassenen, das schließlich mit dem Tod endet. Turrini zieht dafür Vergleiche aus der Bibel heran, besonders den Opfertod von Jesus Christus.

7 Am Beginn des Opfertods steht ein «Letztes Abendmahl», ein gemeinsames Essen von Hans und seinen Arbeitskollegen, die sich gemeinsam eines für die Betriebsleitung vorbereiteten kalten Buffets bemächtigen. In den Worten

Das ist Brot von unserem Fleisch

Wein von unserem Blut

Wir wollen nicht mehr gefressen werden

wir fressen uns selber ${ }^{6}$

spiegelt sich ein verzweifelter Versuch der Arbeiter, die durch Entlassungsmaßnahmen einem immer stärkeren Druck ausgesetzt sind, aufzubegehren. Diese Revolte wird mit Hans' Hinauswurf sanktioniert.

9 Kurze Zeit später ist Hans Ordner im Stahlwerk. Ab dem Moment, in dem er diese Arbeit akzeptiert, geht er zum Klassenfeind über und verrät die Sache der Arbeiter. Aus Jesus aus dem «Letzten Abendmahl» ist Judas geworden, auch wenn sich Hans dessen nicht bewußt ist. Das Verhalten seiner früheren Arbeitskollegen ihm gegenüber machen es ihm aber deutlich. Er wählt schließlich den Tod, um sich einerseits vordergründig - für seinen Verrat selbst zu bestrafen, andererseits sieht Hans keine Überlebensmöglichkeit außerhalb der Arbeitergemeinschaft. Er opfert sich dem «Gott Stahlindustrie» - die Klassensolidarität ist aber gerettet.

Anna erleidet dasselbe Schicksal wie ihr Mann, nach der Entlassung aus der Schuhfabrik nimmt sie eine Stelle als Darstellerin in einem Pornofilm an. Diese Tätigkeit läßt sich anfänglich nicht ohne gewisse Schwierigkeiten ausführen. Während der Kopulation enthüllen die beiden Filmpartner ihre Gründe dafür. Sie erkennen im jeweils anderen den Klassenbruder. Das Mitwirken in einem pornographischen Film wird als Arbeit erkannt und ist als solche moralisch vertretbar.

Anna: Muß man echt stöhnen?

Jugoslawe: Jetzt ist schon Film. Mutti, blasen

Anna: Es geht alles so schnell

Jugoslawe: Ja, schnell. Film ist teuer (...)

Jugoslawe: Jetzt ficken.

Der Arbeiter legt sich auf Anna.

Arbeiter: Schweinemensch. Ich kannnicht.

Anna: Ich auch nicht.

Jugoslawe: Nur Schönes denken. In meiner Heimat, Cres geht die Sonne im Meer unter. Mit dem Boot hinausfahren zur Stelle. In das klare Wasser schauen und die Sonne sehen am Grund. Nur Schönes denken.

Anna: Das Schönste war einmal in meiner Zeit als Küchenhilfe (...) Die Köchin hat mich immer zum Salatwaschen in den Hof geschickt. Im Winter waren die Finger ganz rot. (...) Eines Tages schaute der Baumeister durch das Klofenster und sah mich und den Salat ${ }^{7}$.

Pornographie durchzieht das Stück wie ein Leitmotiv, sie wird sowohl von der Seite des jugoslawischen Produzenten als auch von der des Konsumenten gezeigt. Das Betrachten von Pornofilmen ist Hans' bevorzugte Freizeitgestaltung. Es zieht das passiv apathische Glotzen den Verführungskünsten seiner Frau vor, Ausdruck seines durch die Arbeit völlig verarmten Gefühlslebens. Die Arbeit am Hochofen zerstört jegliche 
Lust und Fähigkeit zu einer kommunikativen Sexualität. Die Kommunikation hat dem Grunzen Platz gemacht.

Provokation ist - wie in jedem Turrini-Stück integrierter Bestandteil seiner Dramaturgie. Sein erklärtes Ziel ist es, «Das Volk...am geliebten Fraß (das Volkstheater) ersticken zu lassen $»^{8}$. Die Zerstörung des traditionellen Volksstücks als Programm seiner Dramaturgie stellt Turrini in eine Reihe mit den Vertretern der klassischen Avantgarde, die - nach Peter Bürger - in der Provokation ein Mittel zur «Liquidierung der Kunst als einer von der Lebenspraxis abgespaltenen Tätigkeit» ${ }^{9}$ sieht. Der Akt der Provokation selbst nimmt die Stelle des Werks ein.

\section{II}

Die Vertreter der Arbeitgeber, mit denen Hans konfrontiert wird - die Personalchefin, ein Quizmaster und der Arbeitsminister - sind anders als die Arbeiter keine real existierenden Personen. Es handelt sich um Allegorien für den Kapitalismus, jede eine andere Facette repräsentierend.

Die erste Konfrontation mit der Kapitalseite hat Hans mit der Personalchef in. Sie macht Hans das verlockende Angebot, gegen eine großzügige Entschädigung freiwillig den Betrieb zu verlassen. Als er sich nach längerem Drängen weigert, legt sie ihr Kleid ab und verwandelt sich in eine peitschenschwingende Lederlady, die ihn auf diese Art überzeugen will.

Sie zeigt gleichzeitig zwei Seiten ihrer Person, eine verführerische und eine schmerzhafte, genauso wie das kapitalistische System. Seine Reize locken, aber die Verführungen müssen mit Schmerz und Erniedrigungen bezahlt werden; in dieser sadomasochistischen Beziehung zwischen Kapitalismus und Werktätigen kann nur ein pervers Veranlagter zu Lustgewinn kommen, woraus die Folgerung, der Kapitalismus sei ein perverses System, nur naheliegt.

16 Die mechanisch-selbstzerstörerische Seite des Kapitalismus, der die Arbeiter zum Opfer fallen, wird vom Quizmaster dargestellt. Nach Hans Entlassung verwandelt sich sein Wohnzimmer in eine Bühne. Er ist Kandidat im Spiel 6 aus $45-45$ brauchen Arbeit, aber nur sechs Stellen sind vorhanden. Die Spielregeln dazu lauten so:

\section{Quizmaster:}

Ich stelle Ihnen eine Reihe von Fragen, und Sie antworten mit Ja oder Nein. Wenn Sie Nein sagen, sind Sie leider ausgeschieden, wenn Sie Ja sagen, bleiben Sie im Rennen. Nach jedem Ja schlagen Sie sich selbst ins Gesicht, nicht zu heftig, es ist ja ein Spiel, aber auch nicht zu zaghaft. Alles auf freiwilliger Basis ${ }^{10}$.

17 Hans ist bereit, auf Fragen des Quizmasters Lohneinbußen und lange Anreisewege in Kauf zu nehmen, seinen Arbeitskollegen und seinen Bruder auszubooten. Keine der Fragen treibt ihn zur Revolte, nach jeder geht er einen schmerzhaften Kompromiß mit sich selbst ein.

Erst als er gegen seine eigene Frau vorgehen soll, ist er fähig, nein zu sagen, der Spuk nimmt ein Ende und eine menschenwürdige Existenz ist im letzten Moment gerettet.

Der dritte Repräsentant der Kapitalseite ist der Arbeitsminister. Er ist der oberste Chef der Stahlindustrie, die in Österreich zur Gänze verstaatlicht ist.

Als Hans ihn mit seinem Problem konfrontiert, versteht er sie nicht oder will er sie nicht verstehen. Anstatt sich um Hans' Problem zu kümmern, läßt er sich von einer 
dicken amerikanischen Sängerin in die Vergangenheit zurückführen. In seinen Visionen sieht er die miserable Situation der Arbeiter im letzten Jahrhundert. Durch die Heraufbeschwörung der menschenunwürdigen Zustände in der Industrie im letzten Jahrhundert soll Hans vor Augen geführt werden, wie sehr sich die Situation verbessert hat.

Ein österreichischer Arbeitsminister ist notgedrungen Sozialdemokrat, also Vertreter der Sozialpartnerschaft und also Vertreter des Kompromisses. Er kann Hans Arbeit besorgen, Hans soll sich aber dafür kompromitieren, dadurch, daß er in der neuen Stellung seine ehemaligen Arbeitskollegen überwachen muß.

Minister: Schau nicht so traurig, mein Freund. Was kann ich tun? Ich bin zu jeder Schandtat bereit. Ach ja, du brauchst eine Arbeit. Sollst du haben. Sollst du haben. (...)

Personalchef (zu Hans): Gratuliere, Sie sind der neue Ordner. Der Teufel weiß, wie Sie es geschafft haben, daß der Minister persönlich für Sie interveniert ${ }^{11}$.

Die von Turrini angewandte Technik der Montage in den Szenen, in denen Vertreter des Kapitals auftreten, ist ein weiteres dramaturgisches Hilfsmittel, eine Schockwirkung $\mathrm{zu}$ erzielen. In allen drei Fällen wechselt die anfangs realistische Situation in einen skurrilen Alptraum. Mit der Verwendung dieser skurrilen Elemente greift Turrini auf Motive des Zaubermärchens eines Ferdinand Raimund zurück, in dessen Sücken die Verwendung von Allegorien wesentlicher Bestandteil sind. Turrini steigert im Gegensatz zu seinem literarischen Vorbild die mit Hilfe der Allegorien dargestellten Bilder ins Unerträgliche, um die früher schon erwähnte Schockwirkung beim Publikum auszulösen. Er verzichtet also keineswegs auf jegliche literarische Tradition, sondern er verfremdet sie, um sie für seine Zwecke einsetzen zu können.

\section{III}

«Kunst in die Lebenspraxis zurückführen ${ }^{12}$, lautet das Postulat der Avantgarde. Peter Bürger untersucht, von dieser Position ausgehend, anhand von drei Elementen der Kunst (Verwendungszweck, Produktion und Rezeption), wann sich ihre Abhebung von der Lebenspraxis vollzogen hat. Nach Bürger geschieht das schon teilweise beim Übergang von der sakralen (mittelalterlichen) zur höfischen Kunst und schließlich vollständig beim Übergang von der höfischen zur bürgerlichen Kunst.

Im Mittelalter dient die sakrale Kunst als Kultobjekt in der Religion, die integrativer Bestandteil des täglichen Lebens des Individuums ist. Kunst wird handwerklichkollektiv produziert und kollektiv rezipiert. Die höfische Kunst hat sich von der sakralen Bindung gelöst, sie ist vor allem Repräsentationsobjekt, dient zur Selbstdarstellung des Fürsten und spiegelt das Leben der höfischen Gesellschaft wider. Die Produktion erfolgt nicht mehr im Kollektiv, sondern individuell. Der Künstler entwickelt dabei auch ein Bewußtsein von der Einzigartigkeit seines Schaffens und hebt sich so von der gesellschaftlichen Integriertheit im Mittelalter ab. Die Rezeption bleibt kollektiv, hat aber keinen sakralen, sondern geselligen Charakter. Die bürgerliche Kunst sieht ihre Aufgabe in der Darstellung des Selbstverständnisses ihrer Klasse. Produktion und Rezeption erfolgen individuell, abgehoben von der Lebenspraxis. Der Leser eignet sich Kunstwerke allein, etwa durch Lesen eines Romans, an. Die Kunst dient - so Habermas ${ }^{13}$ - zur Befriedigung residualer Bedürfnisse, Bedürfnisse, die aus der Lebenspraxis der bürgerlichen Gesellschaft verdrängt sind. 

kann, ist auch ein konkretes Thema von Turrinis Reflexionen. Diese beziehen sich auf das Medium seiner Kunst, aber besonders auf die Rolle des Kunstproduzenten in der Arbeiterklasse - er hinter fragt also seine eigene Rolle. industriellen Produktionsesellschaft in aller Schäfe gestellt. Es soll durch die Gestaltung eines Intellektuellen - im konkreten Fall des Werkbibliothekars Shakespeare - die Konfrontation zwischen Arbeitswelt und Kunst erörtert werden. Shakespeare ist die einzige Gestalt eines Intellektuellen im Stück. Er verdankt seine Stelle einer Kopfverletzung aus dem Krieg. Durch diese Wunde behindert, ist er für keine andere Tätigkeit mehr brauchbar als zur Ausgabe von Büchern, die aber keiner will. Er bleibt auf seinen Büchern sitzen und wird von den Arbeitern nur dann halbwegs ernst genommen, wenn er mit ihnen trinkt. Das wird schlielich auch zu seiner Haupttätigkeit. Er ist immer bereit gegen ein Bier mit bedrängten Arbeitern über seine Freunde, die toten Dichter, die er in leeren Flaschen zu erkennen glaubt («die Flasche Goethe, die Flasche Heine etc.» ${ }^{14}$ ) zu sprechen oder jene mit Ratschlägen zu versorgen.

Der Intellektuelle hat nach der Katastrophe des letzten Weltkriegs, die zu verhindern er nicht imstand war, seinen Platz in der Produktionsgesellschaft definitiv verloren. Er ist von der Basis völlig abgetrennt, aus der Gesellschaft ausgegliedert und verwaltet «ein Meer von Büchern $»^{15}$, die mit der erlebten Wirklichkeit des Betriebsalltags nichts $\mathrm{zu}$ tun haben. Er «taugt nur für wirre Kommentare» ${ }^{16}$, bleibt Beobachter, ohne die Möglichkeit und das Interesse zu haben, in die herrschenden Verhältnisse im Betrieb einzugreifen. Der Künstler ist in seiner Kommunikationsfähigkeit ebenso auf sein soziokulturelles Milieu beschränkt wie der Arbeiter oder der leitende Angestellte. Seine Sprachlosigkeit drückt sich zwar nicht durch akustisch nicht wahrnehmbares Reden aus, sondern durch einen unstrukturierten Wortschwall bar jedes logischen Sinns. Shakespeare kommentiert am Ende des Stücks ausführlich Hans' Selbstmord, macht viele pathetische Worte, die aber keine konkreten Folgen auf sein Verhalten haben. Am Ende «...nimmt er eine Flasche nach der anderen aus der Bierkiste und zerschlägt sie auf seinem Kopf. Er lacht $»^{17}$ und kann nur feststellen, daß es ihm eigentlich glänzend geht.

Die Distanz des Schriftstellers zu den Arbeitern erlebt Turrini immer wieder am eigenen Leib:

Die Kinder der Bauern waren meine Spielkameraden... Heute, wenn ich zu Besuch im Dorf bin, treffe ich ab und zu meine alten Spielkameraden. Sie laden mich auf einen Schnaps ein, klopfen mir, nachdem sie genug getrunken haben, auf die Schulter und schwärmen von unserer gemeinsamen Kindheit.

Ich werde unsicher, merke, daß sich meine Sprache verändert, erzähle von Filmen und Theaterstücken, die ich gerade schreibe, und spüre, wie ich immer noch mit falschen Mitteln um ihre Zuneigung kämpfe ${ }^{18}$.

30 Auch wenn die kulturellen Wurzeln dieselben sind, klaffen die intellektuelle Entwicklung des Schriftstellers und des Arbeiters so weit auseinander, daß der Künstler schließlich unfähig wird, dem Werktätigen in dessen Sprache klarzumachen, daß er sich eigentlich um seine Probleme bemühen will. Die Tragik der unmöglichen 
Kommunikation zwischen Intellektuellem und Werktätigen hat schon Siegfried Kracauer erkannt:

Hundert Berichte aus einer Fabrik lassen sich nicht zur Wirklichkeit der Fabrik addieren, sondern bleiben bis in alle Ewigkeit hundert Fabrikansichten ${ }^{19}$.

31 - und als solche gänzlich praxisfremd.

Der Intellektuelle scheitert in seinem Bemühen, aus Kunst eine neue Lebenspraxis zu organisieren, da dem Kunstschaffenden kein adäquates Ausdrucksmittel zur Verfügung steht, um direkt in den Lebensverhältnissen der Werktätigen wirksam zu werden.

Alles, was er am Ende von sich geben kann, ist schließlich nichts Anderes als Grunzen!

\section{NOTES}

1. - Peter Turrini: Zu Ende mit den Worten der schönen Kunst, In: P. T., Texte, Daten, Bilder, hg. v. W. Schuch und K. Siblewski, Frankfurt/M, Sammlung Luchterhand, 1991, S. 13.

2. - Ende der sechziger, Anfang der siebziger Jahre entstand im bayrischösterreichischen Raum im Zuge eines radikalen Bewutseinswandels, dessen sichtbarer Ausdruck in Österreich der Wechsel der Regierungspartei war, ein «Neues Volksstück». Behandelt werden Themen wie Armut und Außenseitertum trotz Wirtschaftswachstums und Vollbeschäftigung. Zu den wichtigsten Vertretern gehören in Bayern F. X. Kroetz, M. Sperr und R. W. Fassbinder, W. Bauer, P. Turrini und F. Mitterer in Österreich. Ihre Vorbilder sind Ö. v. Horvath und ML. Fleisser.

3. - In der österreichischen Literatur des 20. Jahrhunderts hat Sprache als Thema literarischer Texte eine lange Tradition. Autoren wie Ingeborg Bachmann orientieren sich in den fünfziger und sechziger Jahren an der Sprachskepsis Wittgensteins und prüfen, was Sprache zu leisten vermag. Sprache als Herrschaftsinstrument ist Thema bei P. Handke oder im Antiheimatroman der siebziger und achtziger Jahre.

4. - Peter Turrini: Die Minderleister, Frankfurt/M, Sammlung Luchterhand, 1989, S. 133. In der Folge mit ML zitiert.

5. - Vergleiche dazu Hölderlins Stück «Der Tod des Empedokles», in dem Empe-dokles den Freitod im Vulkan wählt, um das Leben des Gemeinwesens zu erneuern.

6. - ML S. 59.

7. - ML S. $79 \mathrm{f}$.

8. - Peter Turrini: Lesebuch, Wien, Europaverlag 1978, S. 344.

9. - In meinen Ausführungen zur Avantgarde folge ich weitgehenden Thesen Peter Bürgers in: Peter Bürger: Theorie der Avantgarde, Frankfurt/M, edition suhrkamp 1974.

10. - ML S. 69.

11. - ML S. $107 \mathrm{f}$.

12. - Peter Bürger: Theorie der Avantgarde, S. 29.

13. - Vergleiche dazu: Jürgen Habermas: Legitimationsprobleme im Spätkapitalismus, Frankfurt/M, edition suhrkamp 1973, S. 43 f.
14. - ML S. 86.
15. - ML S. 13.
16. - ML S. 61.
17. - ML S. 132. 
18. - Peter Turrini: Biographie des Lesens, in: P. T.: Mein Österreich, Reden, Polemiken, Aufsätze, Frankfurt/M, Sammlung Luchterhand 1988, S. 66.

19. - Siegfried Kracauer: Die Angestellten. Aus dem neuen Deutschland, Frankfurt/M, edition suhrkamp 1971, S. 16.

\section{RÉSUMÉS}

Peter Turrinis Stück «Die Minderleister» ist ein Musterbeispiel für die avantgardistische Erneuerung des österreichischen Volksstücks. Mit Hilfe von Verfahrensweisen der historischen Avantgardebewegung - Montage, Allegorie und Schock - versucht Turrini einem Thema wie der Krise in der Stahlindustrie und den sich daraus ergebenden Konsequenzen beizukommen.

Der durch seine schwere Arbeit schon völlig abgestumpfte Hilfsarbeiter Hans kann nach seiner Entlassung das Dasein nur mehr durch intensiven Pornographiekonsum ertragen. Eine neue Arbeit, die ihn auf die Seite der Arbeitgeber bringt, isoliert ihn von seinen früheren Kollegen; um dieser Misere zu entgehen, wählt er den Selbstmord.

Parallel dazu untersucht Turrini die Rolle des Intellektuellen und kommt zu dem Schluß, daß dieser zwar parasitenhaft von den Werktätigen lebt, ihnen aber in Kristensituationen keine entscheidende Hilfe bringen kann.

La pièce de Peter Turrini «Éléments moins performants » est un bon exemple pour le renouveau avant-gardiste du théâtre populaire autrichien. Avec les techniques de l'avant-garde historique le montage, l'allégorie et le choc - Turrini essaie de traiter le thème de la crise dans l'industrie de l'acier et les conséquences qui en résultent.

Le manœuvre Hand, abruti par son travail très dur, sombre dans la pornographie après avoir été licencié. Il obtient un nouveau travail comme surveillant dans l'aciérie, ce qui l'isole complètement de ses anciens collègues. Pour échapper à cet isolement, il choisit de se suicider. Parallèlement, Turrini examine le rôle de l'intellectuel. Celui-ci vit comme un parasite des ouvriers, mais il n'est pas capable de leur porter secours en situation de crise.

\section{AUTEUR}

\section{ALFRED STRASSER}

Université Charles-de-Gaulle - Lille III 